\title{
Selective loss of RPGRIP1-dependent ciliary targeting of NPHP4, RPGR and SDCCAG8 underlies the degeneration of photoreceptor neurons
}

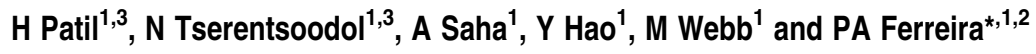

The retinitis pigmentosa GTPase regulator (RPGR) and nephrocystin-4 (NPHP4) comprise two key partners of the assembly complex of the RPGR-interacting protein 1 (RPGRIP1). Mutations in RPGR and NPHP4 are linked to severe multisystemic diseases with strong retinal involvement of photoreceptor neurons, whereas those in RPGRIP1 cause the fulminant photoreceptor dystrophy, Leber congenital amaurosis (LCA). Further, mutations in Rpgrip1 and Nphp4 suppress the elaboration of the outer segment compartment of photoreceptor neurons by elusive mechanisms, the understanding of which has critical implications in uncovering the pathogenesis of syndromic retinal dystrophies. Here we show RPGRIP1 localizes to the photoreceptor connecting cilium (CC) distally to the centriole/basal body marker, centrin-2 and the ciliary marker, acetylated- $\alpha$ tubulin. NPHP4 abuts proximally RPGRIP1, RPGR and the serologically defined colon cancer antigen-8 (SDCCAG8), a protein thought to partake in the RPGRIP1 interactome and implicated also in retinal-renal ciliopathies. Ultrastructurally, RPGRIP1 localizes exclusively throughout the photoreceptor CC and Rpgrip $1^{\text {nmf247 }}$ photoreceptors present shorter cilia with a ruffled membrane. Strikingly, Rpgrip1 ${ }^{\text {nmf247 }}$ mice without RPGRIP1 expression lack NPHP4 and RPGR in photoreceptor cilia, whereas the SDCCAG8 and acetylated- $\alpha$-tubulin ciliary localizations are strongly decreased, even though the NPHP4 and SDCCAG8 expression levels are unaffected and those of acetylated- $\alpha$-tubulin and $\gamma$-tubulin are upregulated. Further, RPGRIP1 loss in photoreceptors shifts the subcellular partitioning of SDCCAG8 and NPHP4 to the membrane fraction associated to the endoplasmic reticulum. Conversely, the ciliary localization of these proteins is unaffected in glomeruli or tubular kidney cells of Rpgrip1 $^{\text {nmf247 }}$, but NPHP4 is downregulated developmentally and selectively in kidney cortex. Hence, RPGRIP1 presents cell type-dependent pathological effects crucial to the ciliary targeting and subcellular partitioning of NPHP4, RPGR and SDCCAG8, and acetylation of ciliary $\alpha$-tubulin or its ciliary targeting, selectively in photoreceptors, but not kidney cells, and these pathological effects underlie photoreceptor degeneration and LCA.

Cell Death and Disease (2012) 3, e355; doi:10.1038/cddis.2012.96; published online 19 July 2012

Subject Category: Neuroscience

Abnormal biogenesis or deregulation of the function of primary cilia is thought to promote a wide variety of clinical manifestations commonly referred as ciliopathies. ${ }^{1,2}$ Yet, the molecular pathogeneses of these diseases are ill-defined, due to our lack of understanding of functional relationships between ciliary components and subcellular processes supporting ciliary targeting. Ciliated photoreceptor neurons present a primary cilium connecting two morphologically and functionally distinct subcellular compartments, the inner and outer segments. ${ }^{3}$ The connecting cilium (CC), which is thought to be equivalent to the transition zone (TZ) of other cilia, projects outward from the basal body (BB) with a membrane-coated bundle of double microtubules organized in a $9+0$ pattern and becomes the axoneme (AX) upon entering the outer segment. ${ }^{3}$ The CC is likely the only route of cargoes' trafficking between inner and outer segment compartments of photoreceptors and is vital to ciliogenesis, genesis of the outer segment and delivery of components for its maintenance. Yet, multiple lines evidence support that ciliary components shared by distinct cell types often perform cell type-specific function(s) for reasons that are not understood. ${ }^{4-11}$

The retinitis pigmentosa GTPase regulator interacting protein 1 (RPGRIP1) interactome was found to be comprised of at least three proteins, RPGR, nephrocystin-4/nephroretinin (NPHP4) and RPGRIP1 itself. ${ }^{12-16}$ Although human-recessive

\footnotetext{
${ }^{1}$ Department of Ophthalmology, Duke University Medical Center, Durham, NC, USA and ${ }^{2}$ Department of Pathology, Duke University Medical Center, Durham, NC, USA ${ }^{*}$ Corresponding author: PA Ferreira, Department of Ophthalmology, Duke University Medical Center, DUEC 3802, 2351 Erwin Road, Durham, NC 27710 , USA. Tel: 919 684 8457; Fax: 919684 3826; E-mail: paulo.ferreira@duke.edu

${ }^{3}$ These authors contributed equally to this work.

Keywords: retinitis pigmentosa GTPase regulator-interacting protein 1 (RPGRIP1); nephrocystin-4 (NPHP4); serologically defined colon cancer antigen 8 (SDCCAG8); retinal-renal ciliopathies; ciliary targeting; protein targeting and sorting

Abbreviations: RPGRIP1, retinitis pigmentosa GTPase regulator-interacting protein 1; RPGR, retinitis pigmentosa GTPase regulator; NPHP4, nephrocystin-4/ nephroretinin; SDCCAG8, serologically defined colon cancer antigen-8; GRP78, glucose-regulated protein of $78 \mathrm{kDa}$; Nup62, nucleoporin 62; GAPDH, glyceraldehyde 3-phosphate dehydrogenase; LCA, Leber congenital amaurosis; SLSN, Senior-Løken syndrome; CRD, cone-rod dystrophy; NPHP, nephronophthisis; RP, retinitis pigmentosa; ER, endoplasmic reticulum; CC, connecting cilium; TZ, transition zone; AX, axoneme; BB, basal bodies; SR, striated rootlets

Received 09.5.12; revised 01.6.12; accepted 21.6.12; Edited by P Salomoni
} 
mutations in RPGRIP1 are associated only to photoreceptor dystrophies typically with early onset and rampant progression, such as Leber congenital amaurosis (LCA6, (MIM 605446, 613826)), ${ }^{17-20}$ cone-rod dystrophy (CRD13 (MIM $608194))^{21}$ and juvenile retinitis pigmentosa, ${ }^{22}$ heterozygous non-synonymous variants of RPGRIP1 and distinct from all photoreceptor dystrophies-causing mutations were found to be associated to various forms of glaucoma. ${ }^{23}$ The exact causes of the clinical and genetic heterogeneity of RPGRIP1 remain unclear, but they likely arise from the differential expression or function of distinct RPGRIP1 isoforms among retinal neurons and species, ${ }^{7,13,24,25}$ and loss-of-function (e.g., LCA) or gain-of-function effects (e.g., glaucoma) of mutations between distinct retinal cell types. In this regard, the largest isoform, RPGRIP $1 \alpha(\sim 175 \mathrm{kDa})$, is expressed specifically in the retina, where it is localized to photoreceptor neurons. ${ }^{7,13,26}$ Distinct domains of RPGRIP1 $\alpha$ interact directly with RPGR ${ }^{13,27}$ and NPHP4. ${ }^{14}$ Human mutations in RPGR, NPHP4 or selective-encoding domains of RPGRIP1 $\alpha$ disrupt specifically the interaction between these partners ${ }^{13,14,27}$ and they cause retinal dystrophies with or without the involvement of extra-ocular organs (e.g. kidney) and with variable clinical manifestations, such as LCA (MIM 605446, 613826), ${ }^{17-20}$ CRD1 ((MIM 608194, 304020)), ${ }^{21,28}$ retinitis pigmentosa alone (RP3 (MIM 300029) $)^{29-31}$ or linked to other systemic clinical presentations (MIM 300455), ${ }^{32}$ X-linked atrophic macular degeneration (MIM 300834), ${ }^{33}$ the retinal-renal dystrophy, Senior-Løken syndrome (SLSN4 (MIM 606996)) ${ }^{32}$ or nephronophthisis (NPHP4 (MIM 606966)). ${ }^{34}$

A dog and two mouse disease models of Rpgrip 1 have been reported with variable phenotypic manifestations as they relate to the suppression of RPGRIP1 expression and/or retinal phenotypic presentations, such as failure to elaborate the outer segment compartment of photoreceptor neurons, disease onset and progression (e.g. rate of photoreceptor degeneration). ${ }^{35-38}$ In this regard, the Rpgrip $1^{\text {nmf247 }}$ mice present loss of expression of RPGRIP1 and they recapitulate well the clinical expression of LCA. ${ }^{35}$ Conversely, mouse and dog disease models of Nphp4, fail to recapitulate selective and hallmark clinical presentations of the human disease, such as renal disease (nephronophthisis), ${ }^{33,34}$ even though photoreceptor degeneration occurs rampantly. ${ }^{5,6}$ The reasons for such conspicuous genotype-phenotype discrepancies among tissues and species are elusive, but emerging evidence support that the subcellular assembly, sorting and targeting of components of pre-ciliary complexes mediated by
RPGRIP1 present remarkable cell type-dependent structural and functional plasticity. ${ }^{27}$ Further, NPHP-5 (SLSN5 (MIM 609237, 609254)), CEP290/NPHP-6 (SLSN6, LCA10 (MIM $610189,610142,611755)$ ) and serologically defined colon cancer antigen-8 (SDCCAG8; SLSN7 (MIM 613524)), whose impairments are also implicated directly in retinal-renal diseases, are additional candidates to partake in the genesis of such ciliary complexes, because they localize to the cilium and may participate dynamically and in a cell type-dependent manner in higher-order assembly complexes with RPGRIP1. ${ }^{39-41}$ To shed light into the selective role(s) of RPGRIP1 in photoreceptor function and degeneration, this report defines the relative ciliary localization of components of the RPGRIP1 interactome in photoreceptor and kidney cell types and it uncovers pivotal physiological roles of RPGRIP1 in the ciliary targeting of NPHP4, RPGR and SDCCAG8 selectively in photoreceptor neurons, but not in various cell types of the kidney.

\section{Results}

RPGRIP1 $\alpha$ determines distinct ciliary localizations of NPHP4, RPGR and SDCCAG8 in photoreceptors. The localizations of RPGRIP $1 \alpha$ and its assembly components at various ciliary regions of photoreceptors (Figures $1 \mathrm{a}$ and $\mathrm{b}$ ) were compared between wild-type and Rpgrip $1^{\text {nmf247 }}$ mice at $\mathrm{P} 12$ of age (Figures $1 \mathrm{c}-\mathrm{g}$ ), when the outer segments begin to develop in wild-type mice and importantly, before pathomorphological changes in the inner segments and cell death ensue in Rpgrip $1^{\text {nmf247 }}$ photoreceptors, ${ }^{35}$ because the outcome of these manifestations may lead to the expression of confounding (secondary) phenotypes. We employed highresolution immunofluorescence microscopy with several antibodies against ciliary markers and components of the RPGRIP1 interactome. RPGRIP1 $\alpha$ localized to the CC distally to the centriole/BB marker, centrin-2, ${ }^{42}$ with which it colocalizes partially (Figure 1c). RPGRIP1 $\alpha$ was absent from the cilium of Rpgrip $1^{\text {nmf247 }}$ mice, but centrin-2 immunolocalization was not affected by the loss of RPGRIP1 $\alpha$ (Figure 1c). RPGRIP1 $\alpha$ also abuts distally another ciliary marker, acetylated $\alpha$-tubulin, but its immunostaining was strongly decreased in Rpgrip $1^{\text {nmf247 }}$ photoreceptors (Figure 1d). NPHP4, a direct partner of RPGRIP $1 \alpha,{ }^{14}$ is reported to localize, like other NPHPs, to the TZ of cilia in Caenorhabditis elegans, ${ }^{43}$ whereas RPGR, another direct partner of RPGRIP1 $\alpha,{ }^{12,13,16}$ and SDCCAG8, also localize to

\footnotetext{
Figure 1 RPGRIP1 $\alpha$ determines distinct ciliary localizations of proteins in mouse photoreceptor neurons. (a) Schematic diagram of a rod photoreceptor with its ciliary region connecting the inner (IS) and outer segment (OS) compartments depicted within the circle (left). Structural organization of the schematic and amplified ciliary region is noted on the right. (b) Overall primary structure of RPGRIP1 $\alpha$ with its domains, protein kinase C conserved region 2 (C2) and RPGR-interacting domain (RID), and its interacting partners, NPHP4 and RPGR, and examined by this study. (c-g) Left and right panels, respectively, are low- and high-magnification images of distal regions of P12 retinas showing: localization of RPGRIP1 $\alpha$ in the connecting cilium and juxtaposed distally to the centriolar/basal body marker, centrin-2, whose localization in the basal body is not affected by loss of RPGRIP1 $\alpha$ in Rpgrip $1^{\text {nmf247 }}$ (c); ciliary localization of RPGRIP1 $\alpha$ distal of and abutting acetylated $\alpha$-tubulin and whose ciliary signal is strongly decreased in Rpgrip $1^{\text {nmf247 }}$ (d); ciliary localization of RPGRIP1 $\alpha$ distal to NPHP4, whose ciliary localization is abolished in Rpgrip ${ }^{\text {nmt247 }}$ (e); localization of RPGR at the connecting cilium distal to NPHP4, the ciliary localization of both proteins are abolished in Rpgrip $1^{\text {nmf247 }}$ (f); NPHP4 ciliary localization abutting proximally SDCCAG8, whose ciliary localization is strongly reduced in Rpgrip $1^{n m f 247}(\mathbf{g})$. Note the localization of NPHP4 extends into the inner segments to the striated rootlets. (h) Quantitation of ciliary proteins from immunoblots of retinal homogenates (left). NPHP4 and SDCCAG8 levels do not change between wild type and Rpgrip ${ }^{\text {nmf247 }}$, whereas acetylated $\alpha$-tubulin and $\gamma$-tubulin are upregulated in Rpgrip 1 ${ }^{n m f 247}$. Bars represent the mean \pm S.D. $(n=4) . P<0.05$ is considered significant. Low- and high-magnification scale bars: 10 and $0.5 \mu$ m; + I+ , wild type; - I - Rpgrip1 ${ }^{\text {nmf247 }}$; AU, arbitrary units; AX, axoneme; BB, basal body; CC/TZ, connecting cilium/transition zone; DC, daughter centriole; Nuc, nucleus; $\mathrm{SR}$, striated rootlet; Syn, synapse. Dash arrows point to locations of schematic regions in photoreceptor neurons in the retinal sections
} 
the mouse cilia. ${ }^{16,25,40}$ In photoreceptors, we found that RPGRIP1 $\alpha$ (Figure 1e), RPGR (Figure 1f) and SDCCAG8 (Figure 1g) abut distally NPHP4 and with RPGRIP1 $\alpha$ presenting partial and lateral ciliary colocalization with NPHP4 at their interface. In contrast, the Rpgrip $1^{\text {nmf247 }}$ cilia were conspicuously void of immunostaining of NPHP4 (Figures 1e-g), whereas immunostaining of SDCCAG8 was extremely weak (Figure 1g). Despite the strong decrease and lack of ciliary staining of SDCCAG8 and NPHP4, respectively, in Rpgrip $1^{\text {nmf247 }}$ photoreceptors, the loss of ciliary a

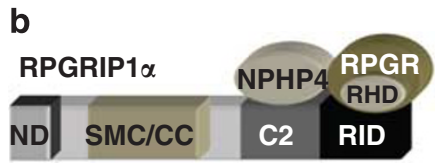

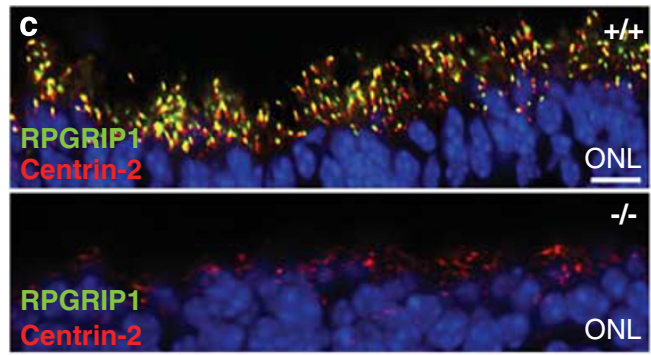
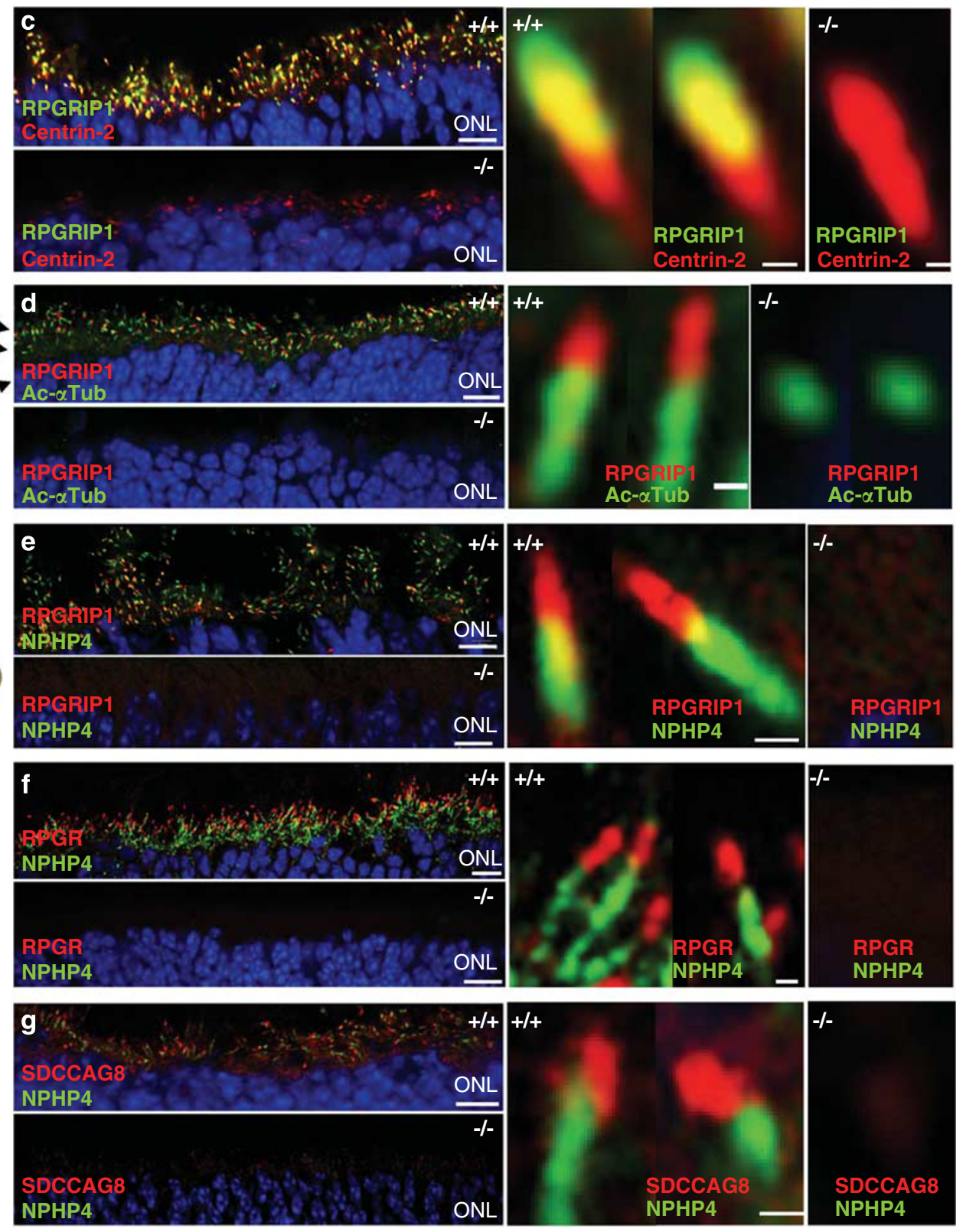

NPHP4
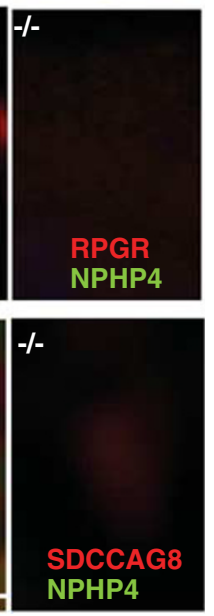
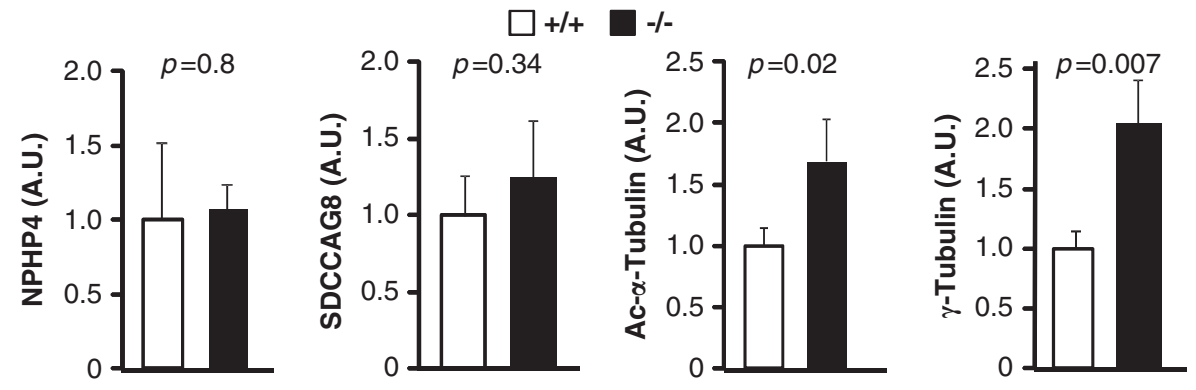
localization of NPHP4 and SDCCAG8 was not accompanied by a decrease of expression of these proteins in Rpgrip $1^{\text {nmf247 }}$ retinas, even though photoreceptor neurons comprise $\sim 70-80 \%$ of all retinal cells (Figure $1 \mathrm{~h}$ ). ${ }^{44}$ Conversely, the expression level of acetylated $\alpha$-tubulin was upregulated significantly in Rpgrip $1^{n m f 247}$ compared with the wild-type retinas, despite of its strong decrease at the photoreceptor cilium of Rpgrip $1^{\text {nmf247 }}$ (Figures 1d and h). Likewise, $\gamma$-tubulin, another microtubule/centriolar marker, was significantly upregulated in Rpgrip $1^{\text {nmf247 }}$ retinas (Figure 1h).

\section{Loss of RPGRIP1 $\alpha$ expression promotes changes in the} subcellular partitioning of SDCCAG8 and NPHP4. The ciliary loss of SDCCAG8 and NPHP4 in Rpgrip $1^{\text {nmf247 }}$ photoreceptors without a parallel decrease of the levels of these proteins in retinal homogenates was surprising, as photoreceptors comprise $\sim 80-70 \%$ of all retinal neurons. ${ }^{44}$ We have previously reported also the expression of a still unknown RPGRIP1 isoform distinct from the photoreceptorspecific RPGRIP $1 \alpha$ isoform in uncharacterized class(es) of amacrine neurons, ${ }^{25,26}$ which collectively account for $\sim 10 \%$ of all murine retinal neurons. ${ }^{45,46}$ Hence, the relative contribution of amacrine neurons to changes in the total level of retinal proteins, if any, upon loss of RPGRIP1, is negligible. We hypothesized that the loss of SDCCAG8 and NPHP4 immunolabeling of Rpgrip $1^{\text {nmf247 }}$ photoreceptors reflect a loss of antigenicity upon impairment of their RPGRIP1-dependent chaperoning, sorting or targeting that may mask or promote the proteolysis of epitopes. This notion is supported by previous findings with transfected cultured cells, where expression of the LCA-causing mutation, $\mathrm{D} 1114 \mathrm{G}$, in the C-terminal RPGR-interacting domain (RID) of RPGRIP1 $\alpha$, promotes the loss of antigenicity of its $\mathrm{N}$-terminal coiled-coil domain toward antibodies against this domain. ${ }^{47}$ Further, RPGRIP $1 \alpha$ has a high propensity to selfaggregate in the absence of its partners, which are critical for the subcellular targeting of RPGRIP $1 \alpha$ to the endoplasmic reticulum (ER) or cytosol. ${ }^{27}$ Hence, to overcome the potential immunohistotochemical limitations described, we examined whether the loss of RPGRIP1 expression promotes a change of the levels of SDCCAG8 and NPHP4 between retinal subcellular fractions (Figure 2). In agreement with our prior studies, ${ }^{26}$ we found that RPGRIP1 $\alpha$ was predominantly found in the cytoskeletal fraction of retinas of wild-type mice (Figures $2 \mathrm{a}$ and b). Although predominant in the cytosolic fraction (cytosolic versus any other fraction, $P<0.016$ ), SDCCAG8 was partitioned between the cytosolic, membrane and nuclear fractions (Figures $2 a$ and $c$ ). NPHP4 was equally distributed between the cytosolic and membrane fractions $(P=0.064)$, where it was largely present (cytosolic and membrane versus any other fraction, $P<0.005)$. Loss of RPGRIP1 $\alpha$ expression in Rpgrip $1^{\text {nmf247 }}$ photoreceptors significantly shifts the localizations of SDCCAG8 and NPHP4 to the ER-associated membrane fraction (Figures $2 a, c, d$ ). These and the observations that RPGR and NPHP4 are critical to the targeting or retrieval of RPGRIP $1 \alpha$ to or from the ER (data not shown), ${ }^{27}$ prompted us to investigate whether the loss of RPGRIP1 $\alpha$ expression promotes ER stress. In comparison to wild-type retinas, the levels of
GRP78 (glucose-regulated protein of $78 \mathrm{kDa}$ ), a stress marker for the ER and cytosol, ${ }^{48,49}$ were significantly increased in Rpgrip $1^{\text {nmf247 }}$ retinas (Figures $2 a$ and e). By contrast, the level of another ER chaperone, calreticulin, ${ }^{50}$ was decreased in Rpgrip $1^{n m f 247}$ retinas, albeit the difference between wild type and Rpgrip $1^{\text {nmf247 }}$ narrowly missed significance $(P=0.053$; Figure $2 \mathrm{a})$.

Heterogeneous subcellular localizations of NPHP4,
RPGR and SDCCAG8 are not affected in Rpgrip1 ${ }^{\text {nmf247 }}$ kidney. To determine whether RPGRIP1 role in the ciliary targeting of NPHP4, RPGR, SDCCAG8 or acetylated $\alpha$-tubulin is cell type and tissue dependent, we examined the ciliary localization of these proteins in the kidney of P21 wildtype and Rpgrip $1^{\text {nmf247 }}$ mice when retinal degeneration is already very advanced. ${ }^{35}$ Acetylated $\alpha$-tubulin and SDCCAG8 partially colocalize to perinuclear centrioles of glomerular and tubular cells of the kidney and such colocalization was not impaired in Rpgrip $1^{\text {nmf247 }}$ mice (Figure 3a). However, there were glomerular and tubular cells in wild-type mice where the centriolar localizations of acetylated $\alpha$-tubulin and SDCCAG8 were mutually exclusive. NPHP4 and RPGR subcellular distributions were heterogeneous between various kidney cell types. NPHP4 localization in the kidney glomerulus was distinct from RPGR (Figure 3b) and SDCCAG8 (Figure 3c). NPHP4 and RPGR had pan-intracellular or centriolar distribution, whereas SDCCAG8 had restricted centriolar localization (Figures $3 \mathrm{~b}$ and c, upper panels). In tubular cells, RPGR was present at discrete foci throughout the cell, the base of the cilium or centrioles (Figure $3 \mathrm{~b}$, middle and lower panels), whereas NPHP4 immunostaining extended from the foot and cap of the $\mathrm{BB}$ distally into the $\mathrm{AX}$ and proximally into the striated rootlet (SR; Figure 3c, lower panel; see the schema in Figure 3d). SDCCAG8 partially colocalized with and flanked NPHP4 at the appendages of the BB in Rpgrip $1^{\text {nmf247 }}$ mice (Figure 3c, lower panel; Figure 3d), but RPGR did not colocalize with NPHP4 (Figure 3b). Further, no changes of NPHP4, SDCCAG8 and $\gamma$-tubulin levels were detected in the cortex and medulla of P12 kidney, but by P21 of age, NPHP4 and $\gamma$-tubulin were downregulated and upregulated, respectively, in the cortex, but not medulla of Rpgrip $1^{\text {nmf247 }}$ (Figure 3e).

Lack of missorting and mistargeting of rhodopsin and M-opsin in Rpgrip $1^{\text {nmf247 }}$ photoreceptors. Ciliary trafficking is thought to have a critical role in the transport of opsins. Even though no evidence supports a direct role of RPGRIP1 in the subcellular targeting of opsins, two prior studies reported the mislocalization of rhodopsin in Rpgrip $1^{\text {tm1Tili }}$ mice, ${ }^{36}$ which still expresses RPGRIP1, but without its C2 and RID domains, ${ }^{35}$ and Rpgrip $1^{\text {nmf247 }}$ mice, which lack RPGRIP1 expression. ${ }^{35}$ One report suggested that opsin mislocalization may arise from the degeneration of the photoreceptors, $^{36}$ regardless of the primary defect as demonstrated by others with unrelated disease mouse models, ${ }^{51}$ whereas the rhodopsin antibody (RET-P1) used by the other study ${ }^{35}$ is known to detect rhodopsin across multiple subcellular compartments of wild-type photoreceptors, including their cell bodies. ${ }^{52}$ Regardless, to distinguish primary from potential secondary phenotypes as they relate 
a

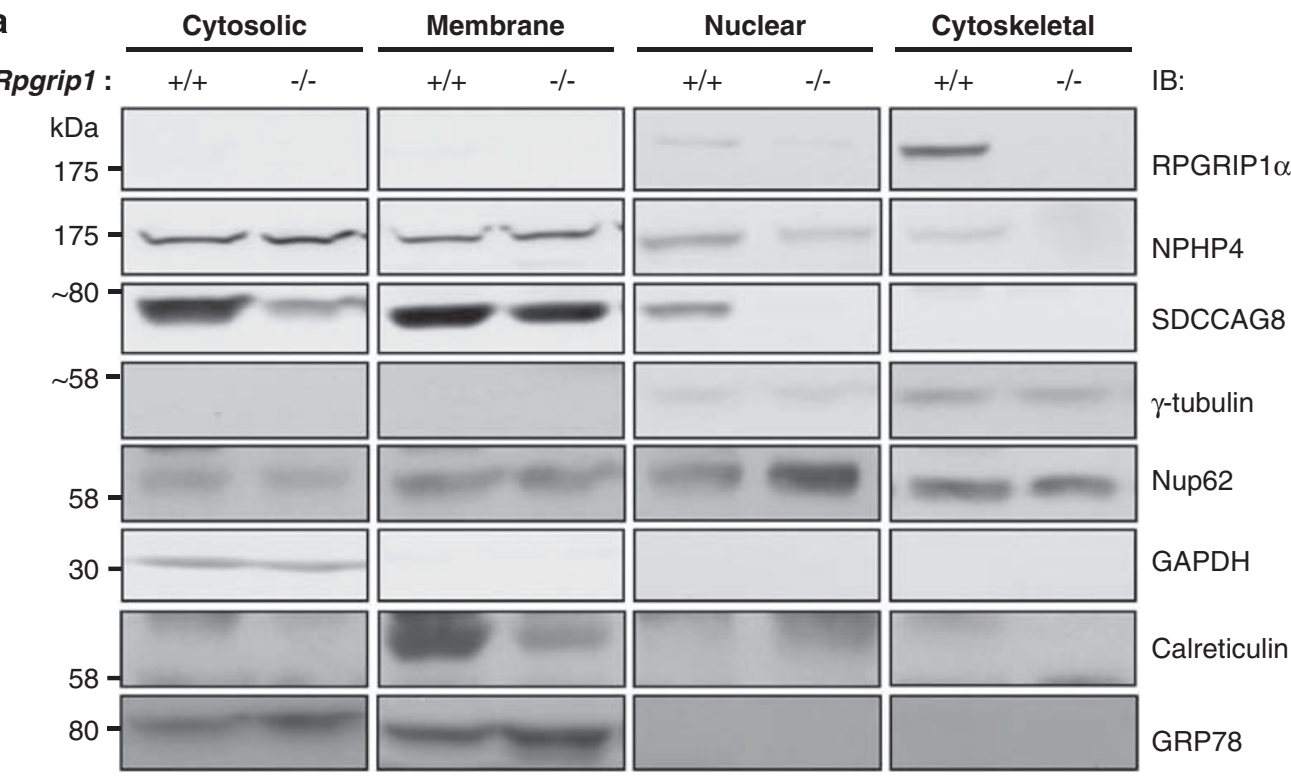

b

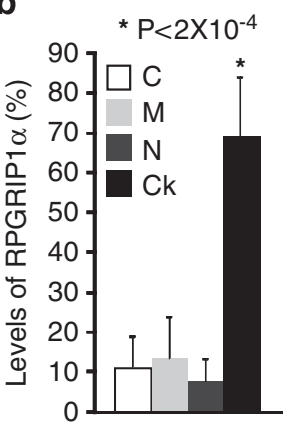

c

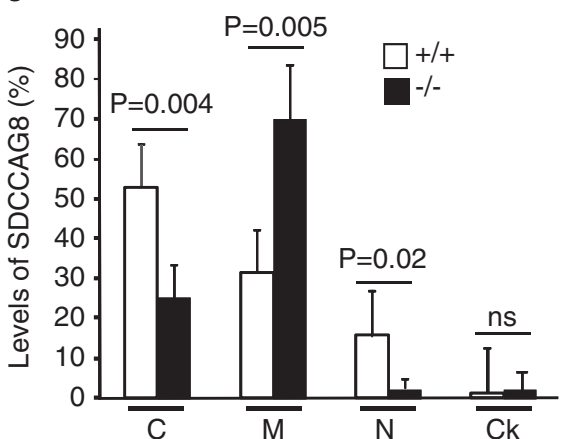

d

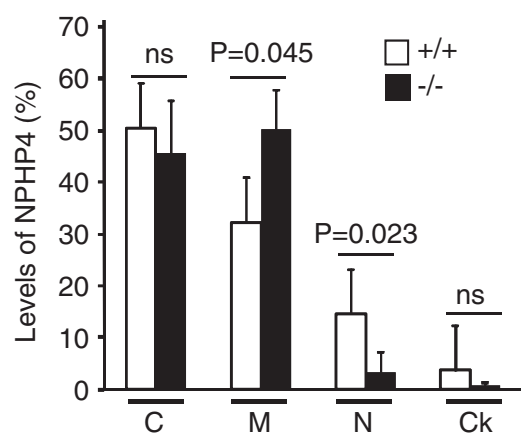

e $\square+/+\square-/-$

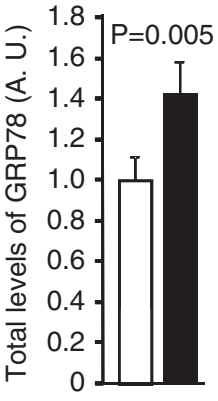

Figure 2 Loss of RPGRIP1 $\alpha$ expression promotes the accumulation of SDCCAG8 and NPHP4 in the retinal membrane subcellular fraction and ER stress. (a) Immunoblots of cytosolic $(\mathrm{C})$, membrane $(\mathrm{M})$, nuclear $(\mathrm{N})$ and cytoskeletal $(\mathrm{Ck})$ subcellular fractions of $\mathrm{P} 14$ retinas of wild-type $(+/+)$ and Rpgrip $1^{\text {nmf247 }}(-/-)$ mice with antibodies against the ciliary proteins, RPGRIP1, NPHP4, SDCCAG8, and the subcellular makers, $\gamma$-tubulin (ciliary/cytoskeleton), Nup62 (pan-subcellular marker), GAPDH (cytosol), calreticulin (ER) and GRP78 (ER and cytosolic stress marker). RPGRIP1 $\alpha$ is mostly in the cytoskeletal fraction, whereas NPHP4 and SDCCAG8 are found mostly in the cytosolic and membrane fractions. The low levels of localization of these components in the nuclear fractions likely represents carry-over of ER membranes connected to the nuclear membrane. In Rpgrip $1^{\text {nmf247 }}$ mice, there is upregulation of careticulin and GRP78. (b) Quantitation of the relative levels of RPGRIP1 $\alpha$ between subcellular retinal fractions. RPGRIP1 $\alpha$ is predominant in the cytoskeletal (Ck) fraction of $+I+$ mice $(n=4,-I-; n=5,+/+)$. (c) Quantitation of the relative levels of SDCCAG8 (c) and NPHP4 (d) between subcellular retinal fractions of wild-type $(+/+)$ and Rpgrip1 $1^{\text {nmf247 }}(-/-)$ mice. SDCCAG8 is localized predominantly in the cytosolic (C) and membrane (M) fractions of $+I+$ mice, whereas its localization increases and decreases in the membrane $(M)$, and cytosolic $(C)$ and nuclear $(N)$ fractions, respectively, of Rpgrip $1^{\text {nmf247 }}$ mice (c). NPHP4 is localized predominantly in the cytosolic (C) and membrane (M) fractions of $+I+$ mice, whereas its localization increases and decreases in the membrane (M) and nuclear (N) fractions, respectively, of Rpgrip $1^{\text {nmf247 }}$ mice (d). (e) Total levels of GRP78 between retinas of wild-type $(+/+)$ and Rpgrip ${ }^{n m f 247}(-/-$ ) mice. There is a $\sim 1.5$-fold increase of GRP78 in retinas of Rpgrip $1^{\text {nmf247 }}(-/-)$ mice. Bars represent the mean \pm S.D. $P<0.05$ was considered significant (Student's $t$-test). $n=4(-/-)$ and $n=5(+/+)$ in (b), (c) and (d); $n=4(-/-,+/+)$ in (e). Nup62, nucleoporin 62; GAPDH, glyceraldehyde 3-phosphate dehydrogenase

to opsin (mis)localization, we re-examined whether the lack of RPGRIP1 expression promotes the mistrafficking of rhodopsin and M-opsin in rod and cone photoreceptors, respectively. First, we employed the same antibodies used by the other studies (e.g., 1D4 and RET-P1) to analyze rhodopsin localization in photoreceptors of P12 and P14 of age when outer segments are beginning to emerge and their growth is well under way, respectively, and importantly, before pathomorphological changes and secondary phenotypes in the inner segments ensue from the absence of RPGRIP1 expression or cell death. No evidence of prominent mislocalization of rhodopsin in the inner segments and cell bodies of rod photoreceptors of Rpgrip $1^{\text {nmf247 }}$ mice of P12 and P14 of age were found with any antibody against rhodopsin (Figure 4a, upper and middle panels). Further, rhodopsin was correctly targeted to the apical end of the inner segments of rod photoreceptors (Figure 4a, upper and middle panels). However, the lack of RPGRIP1 expression was accompanied by a twofold reduction of rhodopsin levels in P12.5 retinas of Rpgrip $1^{\text {nmf247 }}$ compared with the wild-type mice (Figure $4 \mathrm{~b}$ ), an effect that reflects the absence of developing outer segments in Rpgrip $1^{\text {nmf247 }}$ mice. In M-cone photoreceptors, we also did not observe mislocalization and mistargeting of $\mathrm{M}$-opsin and they already appeared much reduced in Rpgrip $1^{\text {nmf247 }}$ mice by P14 of age (Figure 4c). 
a
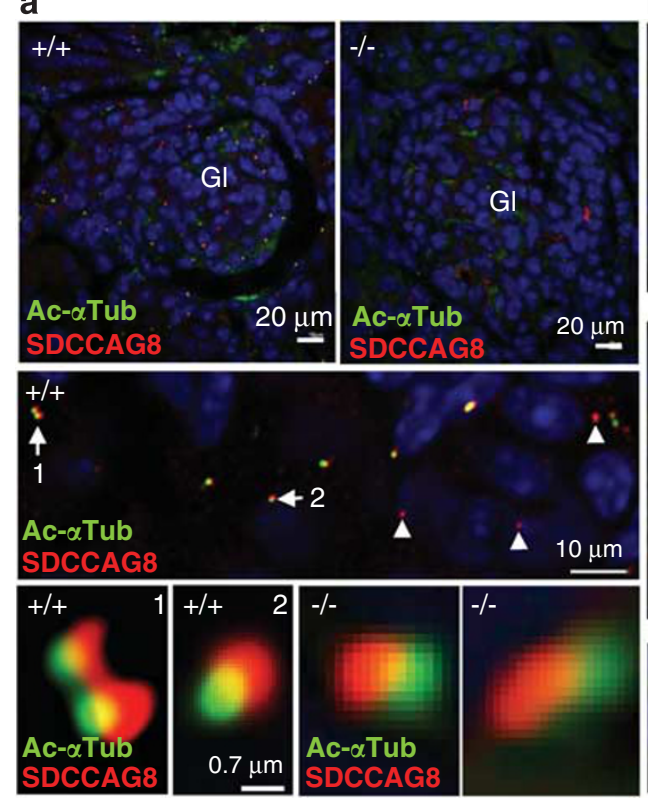

b

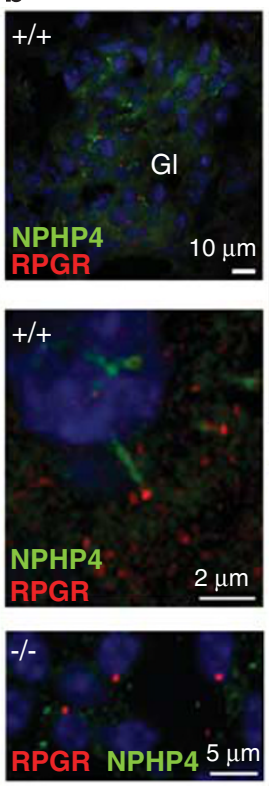

C

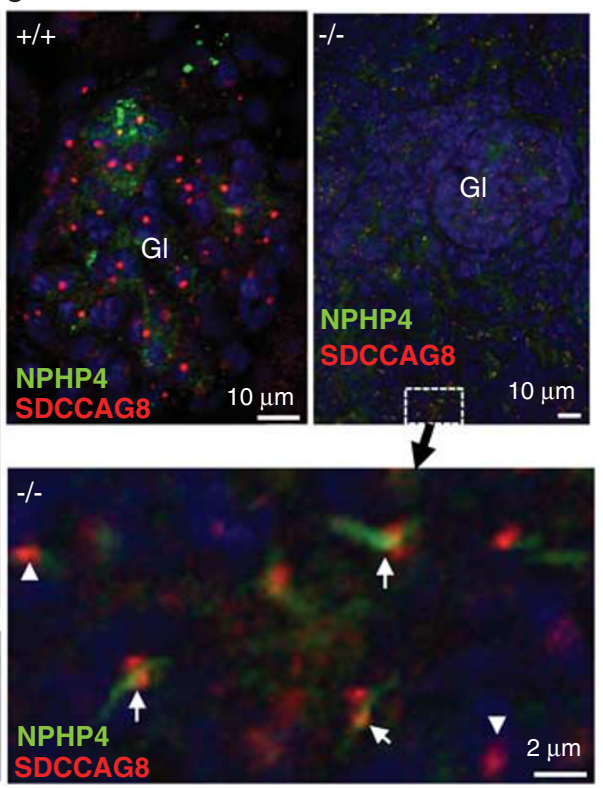

d

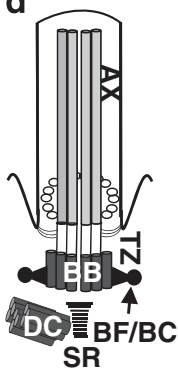

e P12 Cortex
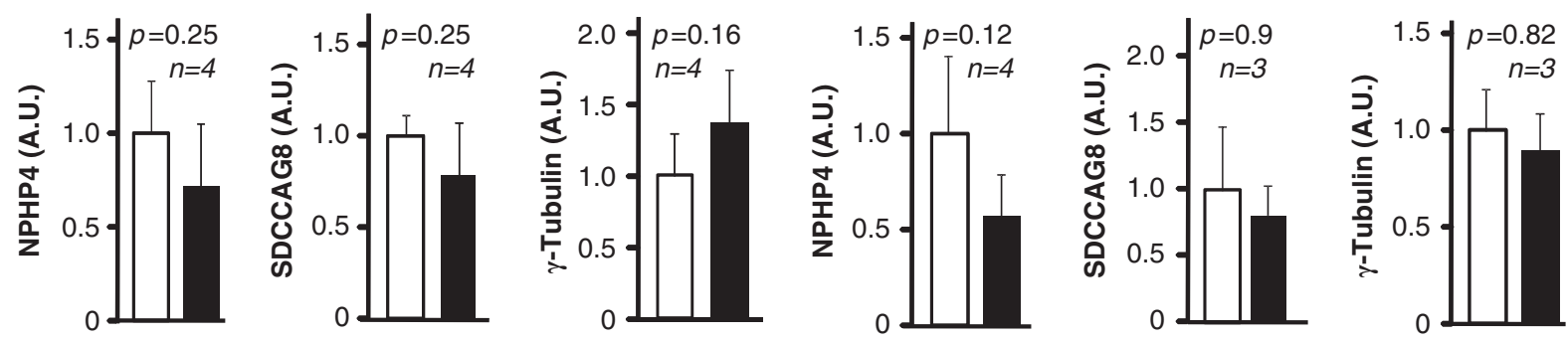

P21 Cortex
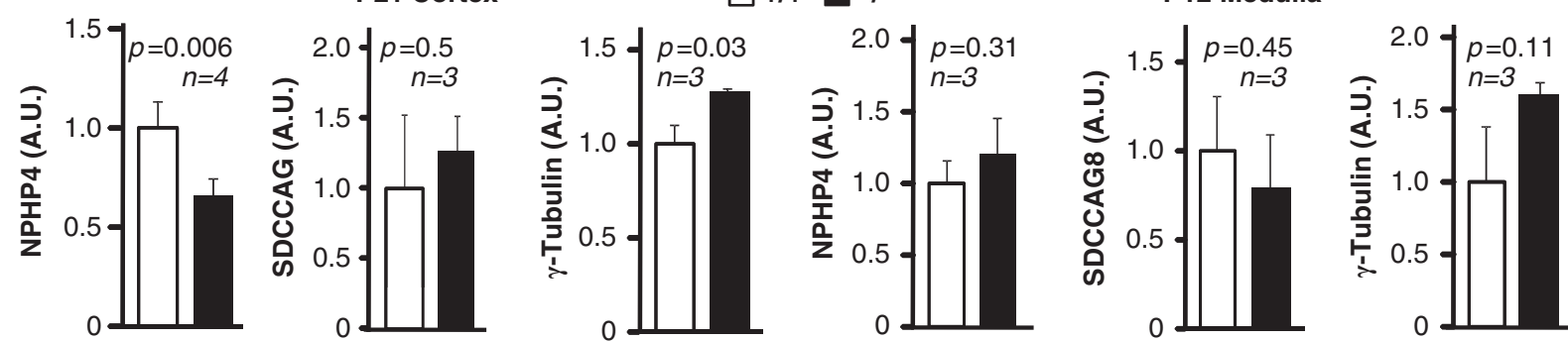

Figure 3 The ciliary/centriolar localization of $\alpha$-tubulin, NPHP4 and SDCCAG8 is unaffected in P21 kidney cells of Rpgrip $1^{\text {nmf247 }}$ mice. Images are low and high magnifications showing: (a) partial colocalization of acetylated $\alpha$-tubulin and SDCCAG8 at the centrioles of glomeruli (Gl) and surrounding kidney tubular cells of wild-type and Rpgrip $1^{\text {nmf247 }}$ mice, the centriolar colocalization is not affected in Rpgrip $1^{\text {nmf247; }}$ (b) NPHP4 and RPGR subcellular distributions varied between glomeruli and tubular cells, their ciliary (middle panel) or centriolar localizations do not overlap (lower panel) in wild-type or Rpgrip $1^{\text {nmf247 }}$ mice; (c) NPHP4 does not colocalize with SDCCAG8 in most glomeruli (Gl) cells of wild type and Rpgrip ${ }^{\text {nmf247 }}$ (upper panels), whereas in tubular cells, the SDCCAG8-stained basal foot (BF) and cap (BC) appendages of the basal body flank laterally the NPHP4-stained axoneme and basal body (lower panel, amplified region of boxed area of upper panel). The partial colocalization of SDCCAG8 and NPHP4 is not affected in Rpgrip $1^{\text {nmf247 }}$. (d) Schematic diagram of a cilium noted on the right. (e) Quantitation of ciliary proteins from immunoblots of medulla and cortex homogenates of kidneys of P12 and P21 of age (left). NPHP4 and $\gamma$-tubulin levels decrease and increase, respectively, in the cortex but not medulla of P21 kidneys. P12 kidneys lack changes in ciliary protein levels between wild type and Rpgrip $1^{n+247}$. Bars represent the mean \pm S.D. $P<0.05$ is considered significant; $P 12, n=4 ; P 21, n=3$. $+/+$, Wild type; $-/-$, Rpgrip $^{\text {nmf247; }}$ AU, arbitrary units; $\mathrm{AX}$, axoneme; $\mathrm{BB}$, basal body; $\mathrm{DC}$, daughter centriole; TZ, transition zone; SR, striated rootlet

Rpgrip $^{\text {nmf247 }}$ photoreceptors exhibit ruffling of the ciliary membrane and shorter cilia. The extent by which disease mutations impair the interplay between ciliary components and the molecular organization underpinning the ultrastructure of the cilium remains unclear. Hence, we examined qualitatively and quantitatively the effects of lack of RPGRIP1 $\alpha$ expression in the ultrastructure of cilia at P12.5 of age, as such effects have not been determined. The developing outer segments of photoreceptors of wild-type mice present disks well stacked and cilia well developed and structured (Figure 5a). Photoreceptors reveal normal BB, $\mathrm{CC}$ and $\mathrm{AX}$ ultrastructures (Figures $5 \mathrm{a}-\mathrm{C}$ ). In contrast, Rpgrip $^{\text {nmf247 }}$ photoreceptors present naked cilia without outer segments and scarce formation of few nascent and 
a
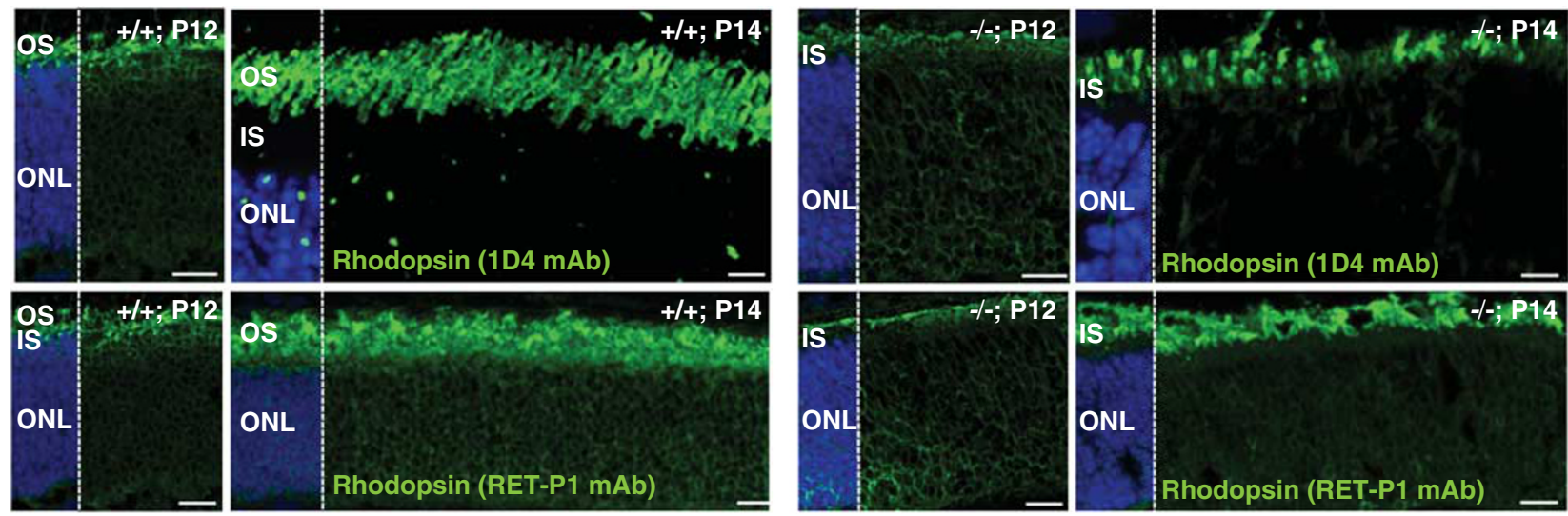

b
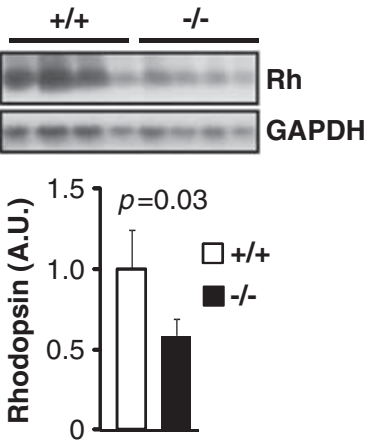

C
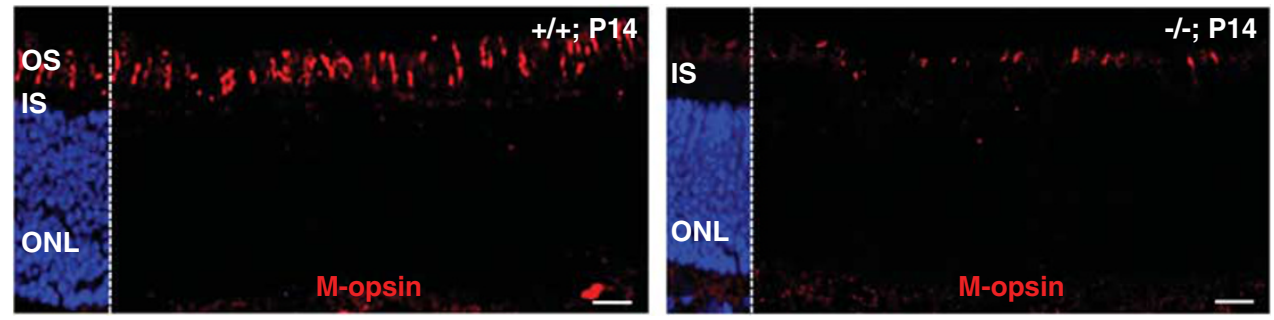

Figure 4 The apical targeting of rhodopsin and M-opsin is unaffected in Rpgrip $1^{\text {nmf247 }}$ photoreceptors. (a) Localization of rhodopsin in rod photoreceptors of wild-type $\left(+/+\right.$, left panels) and Rpgrip $1^{\text {nmf247 }}(-/$ - , right panels) at P12 and P14 of age with two different antibodies against rhodopsin, 1D4 (upper panels) and RET-P1 antibodies (lower panels). Note, the lack of mislocalization or accumulation of rhodopsin in the cell bodies of photoreceptors with any of the antibodies and the correct polarized targeting of rhodopsin to the apical end of the inner segments of rod photoreceptors of Rpgrip ${ }^{\text {nmf247 }}$ mice. In comparison to the 1 D4 antibody, the RET-P1 antibody detects rhodopsin also in the photoreceptor nuclear layer (ONL) of wild-type and Rpgrip $1^{\text {nmf247 }}$ mice. Images were acquired under the same exact acquisition parameters. Duplicated regions stained with DAPI of retinal sections shown are also displayed for reference to the localization of the cell bodies (ONL) of photoreceptors. (b) Qualitative (upper panel) and quantitative (lower panel) immunoblot analyses of rhodopsin levels in retinal homogenates of wild-type and Rpgrip $1^{\text {nmf247 }}$ mice of P12.5 of age. Bars represent the mean \pm S.D. $(n=4) . P<0.05$ is considered significant. (c) Localization of M-opsin in cone photoreceptors of wild-type (left panel) and Rpgrip $1^{\text {nmf247 }}$ mice (right panels) at P14 of age. Note, the lack of mislocalization or accumulation of M-opsin in the cell bodies of cone photoreceptors and the correct polarized targeting of M-opsin to the apical end of the inner segments of cone photoreceptors of Rpgrip $1^{\text {nmf247 }}$ mice. The number of M-cone photoreceptors is also decreased in Rpgrip ${ }^{\text {nmf247 }}$ mice. Images were acquired under the same exact acquisition parameters. Duplicated regions stained with DAPI of retinal sections shown are also displayed for reference to the localization of the cell bodies (ONL) of photoreceptors. Scale bars: $20 \mu \mathrm{m}$ in all panels, except in panels of P14 of age with 1D4 staining, where scale bar is $10 \mu \mathrm{m}$. $+/+$, Wild type; $-/-$, Rpgrip 1 $^{\text {nmf247 }}$; AU, arbitrary units; DAPI, 4',6-diamidino-2-phenylindole; IS, inner segment of photoreceptors; ONL, outer nuclear layer; OS, outer segment of photoreceptors

very irregular disks (Figures $5 d-f$ ). Although the overall morphology and relative positioning of accessory ciliary structures, such as the orthogonal positioning between the BB and daughter centrioles, SR and organization of microtubule doublets, appeared normal, there were two significant ultrastructural abnormalities found in ciliary structures of photoreceptors of Rpgrip $1^{n m f 247}$. First, there was a decrease of the length of the CC and BB by at least by $\sim 25 \%$ compared with age-matched wild-type mice (Figures $5 a, e, I)$. In light of the lack of outer segment disks and a well-defined boundary between the $C C$ and $A X$, the decrease in ciliary length of photoreceptors in Rpgrip $1^{\text {nmf247 }}$ is very likely underestimated. Our computations of the length of Rpgrip $1^{\text {nmf247 }}$ cilia likely include part of the AX (Figure 1a) as they reflect the distances from the base of the $\mathrm{BB}$ up to the longest ciliary tips observed among a large sample of photoreceptors examined. In contrast, the ciliary length of wild-type mice was calculated from the BB to the end of the $\mathrm{CC}$ defined at the level of the base the first nascent disk. Second, Rpgrip $1^{\text {nmf247 }}$ photoreceptors exhibited the ruffling of the ciliary membrane throughout the cilium (Figures $5 g-k$ ), suggesting the weakening of cytoskeletal bridges between the ciliary membrane and cortical cytoskeleton.

The limited resolution of confocal microscopy $(\sim 230 \mathrm{~nm})$ left some ambiguity as to whether the RPGRIP1 $\alpha$ localization to the photoreceptor $\mathrm{CC}$ overlapped partially with that of centrin-2 at the BB (Figure 1c). Hence, we carried out postembedding ultrastructural immunocytochemistry to define precisely the ciliary boundaries of RPGRIP $1 \alpha$. This approach is comparable with procedures employed for confocal microscopy, as it prevented the loss of RPGRIP $1 \alpha$ antigenicity caused by strong fixation conditions required for transmission electron microscopy. As shown by multiple ultrathin sections of photoreceptors, RPGRIP1 localization was solely restricted to the $\mathrm{CC}$, where it was largely localized throughout its ciliary submembrane region (Figures $5 m-p$ ). RPGRIP1 was excluded from the BB and outer segments (AX) of photoreceptors (Figures $5 m-p)$. The accumulation of RPGRIP1 was typically found at the proximal and distal ends 
of the CC, such as where newly nascent disks emerge (Figures $5 \mathrm{~m}-\mathrm{p}$ ). Quantitation of these observations confirmed that the immunogold particles labeled mostly the $\mathrm{CC}$ (Figure 5q).

\section{Discussion}

This work provides compelling evidence that the RPGRIP1 assembly complex presents a strong degree of subcellular
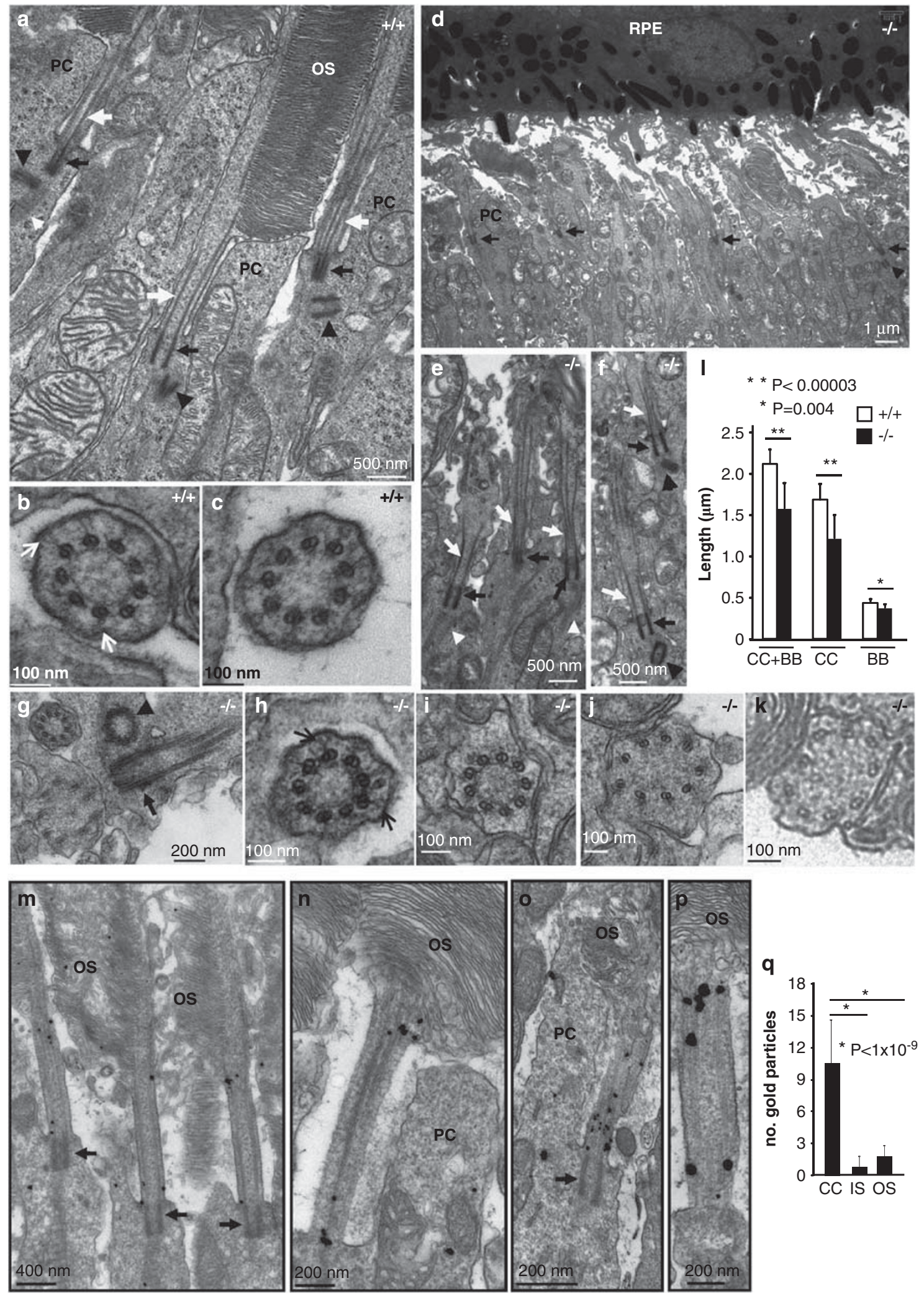
and physiological plasticity. Several conclusions can be drawn from our work. First, SDCCAG8, RPGR and RPGRIP $1 \alpha$ share similar ciliary localization at the $C C$ by abuting distally NPHP4, centrin- 2 and acetylated- $\alpha$-tubulin. Second, RPGRIP1 $\alpha$ determines the cilary localization of NPHP4, RPGR and SDCCAG8 in photoreceptors, because its absence suppresses their ciliary targeting without affecting the overall expression levels of NPHP4 and SDCCAG8, while causing the accumulation of NPHP4 and SDCCAG8 in the ER-associated membrane subcellular fraction. Third, the RPGRIP $1 \alpha$-dependent impairment of the ciliary targeting of NPHP4, RPGR and SDCCAG8 does not affect the apical targeting or cause the missorting of abundant proteins of the outer segment, such as rhodopsin and M-opsin. This observation contradicts a previous report ${ }^{35}$ and the notion that somehow RPGRIP $1 \alpha$ is directly or indirectly implicated in the sorting or targeting of opsins. The reason for this discrepancy arises likely from the broader specificity of the RET-P1 antibody used by Won et al. ${ }^{35}$ In contrast to the 1D4 antibody, RET-P1 is known also to label the cell bodies, fibers and synaptic pedicles of rod photoreceptors and such labeling may cause easily the misinterpretation of data of mutant mouse strains, such as Rpgrip $1^{\text {nmf24752 }} .^{52}$ This limitation can be further compounded with earlier findings that the degeneration of photoreceptors in unrelated genetic mouse models of disease clearly not implicated in protein trafficking promote secondarily the mislocalization of highly abundant photoreceptor proteins, such as opsin. ${ }^{51}$ Regardless, our data support that rhodopsin and M-opsin mislocalizations do not occur before the degeneration of Rpgrip $1^{\text {nmf247 }}$ photoreceptors. Instead, RPGRIP $1 \alpha$ determines the ciliary targeting of critical photoreceptor disease-related proteins (e.g., NPHP4, RPGR, SDCCAG8) that are pivotal to the formation or maintenance of the outer segments of photoreceptors. Fourth, the effects of RPGRIP $1 \alpha$ loss on the ciliary localization of NPHP4, RPGR and SDCCAG8 is highly cell-context dependent, because they are restricted to photoreceptors, but not kidney cells. Further, the ciliary localization of the RPGRIP1 interactome varied between cell types. For example, NPHP4 was excluded from the photoreceptor CC and $A X$, whereas its localization varied in centrioles, $A X$ and/ or TZ of the cilium among cell types of the kidney. This underscores the notion set forth by our prior work that not only the tethering and targeting of the RPGRIP1 interactome harbors structural and functional plasticity and it is highly dynamic at biological level ${ }^{27}$ but such plasticity occurs also physiologically and likely developmentally as shown by this report. Further, these properties of the RPGRIP1 interactome underpin likely the multiple clinical presentations of disease mutations impairing its components.

Finally, it has been unclear whether the RPGRIP1 interactome presents any primary structural ciliary role(s). We have shown previously that the photoreceptor-specific RPGRIP $1 \alpha_{1}$ isoform $^{7}$ is predominantly localized to the detergent-insoluble cytoskeleton fraction of outer segments of bovine photoreceptors. ${ }^{26}$ Such cytoskeleton localization is consistent with the observations herein reported of the localization of the mouse RPGRIP $1 \alpha$ isoform (RPGRIP $\left.1 \alpha_{2}\right)^{7}$ also in the cytoskeletal subcellular fraction and that the loss of ciliary localization of the RPGRIP1 interactome promotes the ruffling of the ciliary membrane likely through subtle changes and weakening of the submembrane cytoskeletal matrix of the CC. Another significant impact of such ciliary loss is the focal abatement of acetylated $\alpha$-tubulin in the proximal cilium, a post-translational modification thought to contribute to the stability of microtubules without affecting the regulation of the length of the cilum. ${ }^{53,54}$ The decrease of acetylated $\alpha$-tubulin at the cilium of Rpgrip $1^{\text {nmf247 }}$ photoreceptors may contribute directly to a decrease of the length of the BB and CC by affecting the transport of crucial cargoes (e.g., SDCCAG8, NPHP4, RPGR) required for the formation of outer segments. Indeed, acetylation of $\alpha$-tubulin is critical to the regulation of transport processes mediated by microtubule-based motor proteins and its misregulation underlies neurodegenerative diseases. ${ }^{55-57}$ It is noteworthy also that the loss of NPHP4 at the $\mathrm{TZ}$ is tolerated by developing cilia in $C$. elegans, but these cilia present ultrastructural cilary defects, such as B-tubules seam breaks, mislocalization of selective ciliary components and deficits in intraflagellar transport. ${ }^{43}$ Yet, our study did not find any abnormal ciliary microtubule structures caused by the lack of RPGRIP1-dependent ciliary localization of NPHP4 in the mouse, another evidence of the high degree of molecular plasticity of the RPGRIP1 complex in ciliary targeting, function or between species. This observation is also in agreement with data that mice lacking NPHP4 do not present abnormal microtubule structures in cilia of photoreceptors and spermatozoa. ${ }^{5}$ Hence, parsing the molecular bases of the degree of functional plasticity conferred by the remodeling of the RPGRIP1 complex and interplay between its partners will provide insights into shared and unique roles the RPGRIP1 components in sorting and targeting processes and pathways among ciliated cell types (e.g., photoreceptors and kidney), species and development of cell type-restricted clinical manifestations.

Figure 5 Ultrastructural ciliary changes and localization of RPGRIP1 $\alpha$ in photoreceptors. Transmission electron micrographs of photoreceptors and their cilia in P12.5 wild type $(+/+)(\mathbf{a}-\mathbf{c})$ and Rpgrip1 $1^{\text {nf247 }}(-/-)$ mice (d-k). (a) Longitudinal section of ciliary regions of rod photoreceptors. (b-d) Transverse sections of the connecting cilium at the distal level of transition zone with an amorphous disk structure and ciliary necklace. (d-f) Low and high magnifications of longitudinal sections of photoreceptors of Rpgrip $1^{\text {nmf247 }}$ mice. Note, Rpgrip $1^{\text {nmf247 }}$ cilia are naked of outer segments. (g-k) Proximal to distal transverse sections of the connecting cilium depicting the ruffling of the ciliary membrane. (I) Quantitative analyses of the length of basal bodies (BB) and connecting cilia (CC) between wild-type and Rpgrip $1^{\text {nmm247 }}$ photoreceptors with the latter having shorter cilia. Bars represent the mean \pm S.D. $(n=21,-I-; n=11,+/+$ from three mice of each genotype). $P<0.05$ was considered significant (Student's $t$-test). Only the longest cilia observed in Rpgrip $1^{n m f 247}$ mice from the sampling of a large number of cilia were included for quantitation analysis. ( $\left.m-p\right)$ Representative ultrastructural images of the restricted immunogold localization of RPGRIP1 $\alpha$ throughout the connecting cilium (CC) of photoreceptors. RPGRIP1 $\alpha$ was excluded from the basal body (arrow) and outer segment/axoneme of photoreceptors. RPGRIP1 $\alpha$ localization was prominent beneath the ciliary membrane (e.g. $\mathbf{m}, \mathbf{p})$ and at the proximal and distal regions of the connecting cilium (e.g. n-p). (q) Quantitation of immunogold particles of RPGRIP1 $\alpha$ in the connecting cilium (CC), inner (IS) and outer segments (OS) of photoreceptors. The majority of immunogold particles are restricted to the connecting cilium (CC). Bars represent the mean \pm S.D. $(n=22) P<0.05$ was considered significant (Student's $t$-test). White arrow, connecting cilium; black arrow, basal body; black arrowhead, proximal (daughter) centriole; white arrowhead, striated rootlets. OS, outer segments of rod photoreceptors; PC, periciliary ridge; RPE, retina pigment epithelium 


\section{Materials and Methods}

Mice. Rpgrip $1^{\text {nmf247 }}$ mice were kindly provided by Patsy Nishina at the Jackson Laboratory (Bar Harbor, ME, USA), ${ }^{35}$ and wild-type mice were on a C57BI6 background and housed in a temperature-controlled and pathogen-free transgenic barrier facility under a 12:12 h light-dark cycle. Animal protocols were approved by the IACUC of the Duke University.

Immunochemistry reagents. Rabbit antibodies (RPGR no. 1, RPGR no. 2) against the recombinant RCC1-homologous domain (RHD) of human RPGR (N-RPGR, a.a.: 1-401) were produced upon four booster shots with recombinant protein $(\sim 100 \mu \mathrm{g})$ and Hunter's TiterMax Gold adjuvant (CytRx Corp., Norcross, GA, USA) followed by affinity-purification against the same antigen under nondenaturing conditions according to the manufacturer's instructions (Stereogene, Arcadia, CA, USA). RPGR no. 2 antibody was employed at $5 \mu \mathrm{g} / \mathrm{ml}$ for immunofluorescence. Other antibodies used for immunofluorescence (IF) or immunoblots (IB) were as following: anti-RPGRIP1 (Ab no. 38, $10 \mu \mathrm{g} / \mathrm{ml}){ }^{26}$ antiNPHP4 (1:100 (IF), 1:1000 (IB)) was kindly donated by Ronald Roepman; ${ }^{14}$ monoclonal mouse anti-acetylated $\alpha$-tubulin $(1: 200$ (IF), 1:25000 (IB), Sigma-Aldrich, St. Louis, MO, USA); anti-SDCCAG8 (1:1000 (IF, IB), Proteintech Group, Chicago, IL, USA); anti-centrin-2 (Cetn-2.4; 1:50) was kindly donated by Jeffrey Salisbury; anti-red opsin no. $21069\left(1: 500\right.$, (IF)) ${ }^{58}$ anti-rhodopsin 1D4 (1:500 (IF), 1:40000 (IB); Millipore, Temecula, CA, USA); anti-rhodopsin RET-P1 (1 $\mu \mathrm{g} / \mathrm{ml}($ IF); Leico Technologies, St. Louis, MO, USA); polyclonal rabbit anti- $\gamma$-tubulin antibody (1:1000 (IB), Abcam, Cambridge, MA, USA); polyclonal rabbit anti-GAPDH (glyceraldehyde 3-phosphate dehydrogenase) antibody (1:500 (IB), Santa Cruz Biotechnology, Inc., Santa Cruz, CA, USA); polyclonal rabbit antiGRP78 (1: 1000 (IB), Thermo Scientific, Rockford, IL, USA); polyclonal rabbit anticalreticulin (1:1000 (IB), Thermo Scientific); monoclonal mouse mAb414 against nuclear pore complex proteins (e.g., nucleoporin 62 (Nup62; $400 \mathrm{ng} / \mathrm{ml} \mathrm{IB),}$ Covance, Emeryville, CA, USA). Alexa Fluor-conjugated secondary antibodies and Hoechst 33342 were from Invitrogen (Grand Island, NY, USA).

Immunohistochemistry and confocal microscopy. Fresh eyes and kidneys of P12.5 and P21 mice were employed for ciliary localizations of proteins, whereas rhodopsin and M-opsin were localized in fixed retinas. Fixation of eyes was carried in $2 \%$ paraformaldehyde, phosphate buffer, $\mathrm{pH} 7.4$, at $4{ }^{\circ} \mathrm{C}$ for $4 \mathrm{~h}$ followed by equilibration in $5 \%$ sucrose, $1 \times \mathrm{PBS}, \mathrm{pH} 7.4$ for $1 \mathrm{~h}$ and then $30 \%$ sucrose, $1 \times \mathrm{PBS}, \mathrm{pH} 7.4$, overnight at $4{ }^{\circ} \mathrm{C}$. Fresh and fixed tissues were frozen in OCT medium (Sakura, Torrance, CA, USA) on dry ice and stored at $-80^{\circ} \mathrm{C}$. Thick sections of $12 \mu \mathrm{m}$ were cut with a microtome (Microm HM550, Walldorf, Germany). Fixed sections were incubated first with $5 \%$ normal goat serum in $1 \times$ PBS, $\mathrm{pH} 7.4,0.1 \%$ Triton $\mathrm{X}-100$, for $1 \mathrm{~h}$, then overnight with primary antibodies in the same buffer followed by secondary antibodies $(2.5 \mu \mathrm{g} / \mathrm{ml})$ in $1 \times$ PBS, $\mathrm{pH} 7.4$ for $1 \mathrm{~h}$. For fresh sections, primary and secondary antibodies were incubated with $0.1 \%$ saponin, $1 \times$ PBS, pH 7.4. Sections were counter-stained with Hoechst $33342(10 \mu \mathrm{g} / \mathrm{ml})$. Z-stacks confocal images $\sim 0.5 \mu \mathrm{m}$ apart, $\sim 5 \mu \mathrm{m}$ deep and with same laser power between wild-type and mutant mice were captured with a Nikon C1-laser scanning confocal/Nikon Eclipse 90i microscope controlled by the EZ-C1 v3.10 software (Nikon, Melville, NY, USA), collapsed, imported to and cropped in Adobe Photoshop CS3.

Transmission electron microscopy, pre-embedding and immunogold labeling. Transmission electron microscopy was performed as described earlier. ${ }^{59}$ Briefly, wild-type and Rpgrip $1^{\text {nmf247 }}$ eyes were fixed in $2 \%$ glutaraldehyde $/ 2 \%$ paraformaldehyde in $0.1 \mathrm{M}$ cacodylate buffer, $\mathrm{pH} 7.2$ at $4{ }^{\circ} \mathrm{C}$ overnight and $4 \%$ glutaraldehyde in $0.1 \%$ cacodylate buffer, $\mathrm{pH} 7.2$ at $4{ }^{\circ} \mathrm{C}$ for $4 \mathrm{~h}$, respectively. Posterior eyes were then post-fixed in $1 \%$ osmium tetraoxide/ $/ .1 \mathrm{M}$ cacodylate buffer and embedded in Spurr resin. Thick sections of $65 \mathrm{~nm}$ were cut with an ultramicrotome (Leica, Ultracut S, Nijmegen, The Netherlands) and stained with $2 \%$ uranyl acetate and $4 \%$ lead citrate. Specimens were visualized with JEM1400 transmission electron microscope (JEOL, Tokyo, Japan) coupled with an ORIUS 1000CCD camera. For immunogold labeling of RPGRIP1, eyes were briefly fixed in $1 \%$ paraformaldehyde for $30 \mathrm{~min}$ at $4{ }^{\circ} \mathrm{C}$ and posterior eyecups embedded in $5 \%$ agarose in $1 \times$ PBS, pH 7.4. Thick eyecup sections of $100 \mu \mathrm{m}$ were obtained with a Leica VT1200S vibratome, blocked in $5 \%$ goat serum $/ 0.2 \%$ Triton X-100, incubated with Ab no. $38(10 \mu \mathrm{g} / \mathrm{ml})$ in $0.2 \%$ Triton X-100/1 $\times$ PBS, $\mathrm{pH} 7.4$ at $4{ }^{\circ} \mathrm{C}$ for $3-4$ days, washed thrice in $1 \times \mathrm{PBS}, \mathrm{pH} 7.4(20 \mathrm{~min})$ and incubated with secondary antibody conjugated with $1.4 \mathrm{~nm}$ nanogold particles $(1: 50)$ (Nanoprobes) at room temperature for $3 \mathrm{~h}$. Sections were washed in $1 \times$
PBS, $\mathrm{pH} 7.4$, post-fixed in $2 \%$ glutaraldehyde in $1 \times \mathrm{PBS}, \mathrm{pH} 7.4$ at room temperature $(10 \mathrm{~min})$, washed in distilled water followed by the enhancement of nanogold particles with $\mathrm{HQ}$ silver enhancement kit (Nanoprobes, Stony Brook, NY, USA). After rinsing several times with $0.1 \mathrm{M}$ cacodylate buffer, $\mathrm{pH} 7.2$, the sections were post-fixed in 1\% osmium tetraoxide, dehydrated in ethanol, infiltrated in Spurr resin, $65 \mathrm{~nm}$ thick sections were cut with an ultramicrotome (Leica Ultracut S), stained with $2 \%$ uranyl acetate and $4 \%$ lead citrate, and imaged by transmission electron microscopy as described.

Tissue homogenates, subcellular fractionation, immunoblot and densitometry analyses. Tissue homogenates from retinas and portions of the medulla and cortex of kidneys were SDS-solubilized, resolved on SDS-PAGE, blotted onto PVDF and incubated with respective antibodies as described earlier. ${ }^{26}$ Immunoblots were scanned and protein quantitation determined with Metamorph v7.7 (Molecular Devices, Sunnyvale, CA, USA) by computing the integrated density values (idv) of non-saturating bands corrected against the background. Corrected idv of each protein was normalized against the corrected idv of GADPH. Relative protein optical densities were plotted with Excel (Microsoft). Statistical analysis was determined by the two-sample Student's $t$-test (assuming unequal variances) with Origin 8.5 (OriginLab, Northampton, MA, USA). The cytosolic, membrane, nuclear and cytoskeleton subcellular fractionations of two frozen retinas of a mouse of P14 of age were prepared with the Qproteome Cell Compartment kit and exactly as described by the manufacturer instructions (Qiagen, Valencia, CA, USA) with the exception that the cytosolic fraction was collected from retinal total lysate upon centrifugation at 1000 r.p.m. All subcellular fractions were solubilized in SDS-sampler buffer, resolved by SDS-PAGE and immunoblots were carried out with antibodies against proteins of interest and markers to each subcellular fraction. The idv of the immunoblot bands were first corrected by subtracting the idv background of the same area in the corresponding lane. Then, they were normalized to the idv of Nup62 of the same fraction, a nuclear pore protein that copurifies with all subcellular fractions ${ }^{60}$ and without any significant changes between wild type and Rpgrip ${ }^{\text {nmf247 }}$.Upon normalization, the idv of all four fractions for each of protein in wild type and Rpgrip $1^{\text {nmf247 }}$ were transformed into a percentage scale (total protein $=100 \%$ ). Average values obtained for each fraction of wild type and Rpgrip $1^{\text {nmf247 }}$ were compared using two-sample $t$-test with assumption of unequal variance at the minimum significance level of 0.05 .

\section{Conflict of Interest}

The authors declare no conflict of interest.

Acknowledgements. We thank P Nishina, J Salisbury and Ronald Roepman for providing the Rpgrip $1^{\text {nmf247 }}$ mice, Cetn-2.4 and NPHP4 antibodies, respectively. Grant support provided from the National Institutes of Health to PAF (EY019492, GM083165), 2P30-EY005722 and Research to Prevent Blindness. PAF is the Jules and Doris Stein Research to Prevent Blindness Professor.

1. Goetz SC, Anderson KV. The primary cilium: a signalling centre during vertebrate development. Nat Rev Genet 2010; 11: 331-344.

2. Fliegauf $M$, Benzing $T$, Omran $H$. When cilia go bad: cilia defects and ciliopathies. Nat Rev Mol Cell Biol 2007; 8: 880-893.

3. Besharse JC, Horst CJ. The photoreceptor connecting cilium, a model for the transition zone. In: Bloodgood RA (ed) Ciliary and Flagellar Membranes New York, NY, 1990, pp 389-417.

4. Hildebrandt F, Zhou W. Nephronophthisis-associated ciliopathies. J Am Soc Nephrol 2007; 18: 1855-1871.

5. Won J, Marin de Evsikova C, Smith RS, Hicks WL, Edwards MM, Longo-Guess C et al. NPHP4 is necessary for normal photoreceptor ribbon synapse maintenance and outer segment formation, and for sperm development. Hum Mol Genet 2011; 20: 482-496.

6. Wiik AC, Wade C, Biagi T, Ropstad EO, Bjerkas E, Lindblad-Toh $\mathrm{K}$ et al. A deletion in nephronophthisis 4 (NPHP4) is associated with recessive cone-rod dystrophy in standard wire-haired dachshund. Genome Res 2008; 18: 1415-1421.

7. Ferreira PA. Insights into X-linked retinitis pigmentosa type 3 , allied diseases and underlying pathomechanisms. Hum Mol Genet 200514 Spec No. 2. R259-67.

8. Fliegauf M, Horvath J, von Schnakenburg C, Olbrich H, Muller D, Thumfart $\mathrm{J}$ et al. Nephrocystin specifically localizes to the transition zone of renal and respiratory cilia and photoreceptor connecting cilia. J Am Soc Nephrol 2006; 17: 2424-2433.

9. Arts HH, Doherty D, van Beersum SE, Parisi MA, Letteboer SJ, Gorden NT et al. Mutations in the gene encoding the basal body protein RPGRIP1L, a nephrocystin- 4 interactor, cause Joubert syndrome. Nat Genet 2007; 39: 882-888.

10. Delous M, Baala L, Salomon R, Laclef C, Vierkotten J, Tory $K$ et al. The ciliary gene RPGRIP1L is mutated in cerebello-oculo-renal syndrome (Joubert syndrome type B) and Meckel syndrome. Nat Genet 2007; 39: 875-881. 
11. Bergmann C, Fliegauf M, Bruchle NO, Frank V, Olbrich $\mathrm{H}$, Kirschner $\mathrm{J}$ et al. Loss of nephrocystin-3 function can cause embryonic lethality, Meckel-Gruber-like syndrome situs inversus, and renal-hepatic-pancreatic dysplasia. Am J Hum Genet 2008; 82: 959-970.

12. Boylan JP, Wright AF. Identification of a novel protein interacting with RPGR. Hum Mol Genet 2000; 9: 2085-2093.

13. Roepman R, Bernoud-Hubac N, Schick DE, Maugeri A, Berger W, Ropers $\mathrm{HH}$ et al. The retinitis pigmentosa GTPase regulator (RPGR) interacts with novel transportlike proteins in the outer segments of rod photoreceptors. Hum Mol Genet 2000; 9 : 2095-2105

14. Roepman R, Letteboer SJ, Arts HH, van Beersum SE, Lu X, Krieger E et al. Interaction of nephrocystin-4 and RPGRIP1 is disrupted by nephronophthisis or Leber congenital amaurosis-associated mutations. Proc Natl Acad Sci USA 2005; 102: 18520-18525.

15. Roepman $R$, Schick D, Ferreira PA. Isolation of retinal proteins that interact with retinitis pigmentosa GTPase regulator by interaction trap screen in yeast. Methods Enzymol 2000; 316: 688-704.

16. Hong DH, Yue G, Adamian M, Li T. Retinitis pigmentosa GTPase regulator (RPGRr)interacting protein is stably associated with the photoreceptor ciliary axoneme and anchors RPGR to the connecting cilium. J Biol Chem 2001; 276: 12091-12099.

17. Dryja TP, Adams SM, Grimsby JL, McGee TL, Hong DH, Li T et al. Null RPGRIP1 alleles in patients with Leber congenital amaurosis. Am J Hum Genet 2001; 68: 1295-1298.

18. Gerber S, Perrault I, Hanein S, Barbet F, Ducroq D, Ghazi I et al. Complete exon-intron structure of the RPGR-interacting protein (RPGRIP1) gene allows the identification of mutations underlying Leber congenital amaurosis. Eur J Hum Genet 2001; 9: 561-571.

19. Hanein S, Perrault I, Gerber S, Tanguy G, Barbet F, Ducroq D et al. Leber congenita amaurosis: comprehensive survey of the genetic heterogeneity, refinement of the clinical definition, and genotype-phenotype correlations as a strategy for molecular diagnosis. Hum Mutat 2004; 23: 306-317.

20. Galvin JA, Fishman GA, Stone EM, Koenekoop RK. Evaluation of genotype-phenotype associations in Leber congenital amaurosis. Retina 2005; 25: 919-929.

21. Hameed A, Abid A, Aziz A, Ismail M, Mehdi SQ, Khaliq S. Evidence of RPGRIP1 gene mutations associated with recessive cone-rod dystrophy. J Med Genet 2003; 40: 616-619.

22. Booij JC, Florijn RJ, ten Brink JB, Loves W, Meire F, van Schooneveld MJ et al. Identification of mutations in the AIPL1, CRB1, GUCY2D, RPE65, and RPGRIP1 genes in patients with juvenile retinitis pigmentosa. J Med Genet 2005; 42: e67.

23. Fernandez-Martinez L, Letteboer S, Mardin CY, Weisschuh N, Gramer E, Weber BH et al. Evidence for RPGRIP1 gene as risk factor for primary open angle glaucoma. Eur $\mathrm{J}$ Hum Genet 2011; 19: 445-451.

24. Lu X, Ferreira PA. Identification of novel murine- and human-specific RPGRIP1 splicevariants with distinct expression profiles and subcellular localization. Invest Ophthalmol Vis Sci 2005; 46: 1882-1890

25. Mavlyutov TA, Zhao H, Ferreira PA. Species-specific subcellular localization of RPGR and RPGRIP isoforms: implications for the phenotypic variability of congenital retinopathies among species. Hum Mol Genet 2002; 11: 1899-1907.

26. Castagnet $P$, Mavlyutov T, Cai Y, Zhong F, Ferreira P. RPGRIP 1s with distinct neuronal localization and biochemical properties associate selectively with RanBP2 in amacrine neurons. Hum Mol Genet 2003; 12: 1847-1863.

27. Patil $\mathrm{H}$, Guruju MR, Cho K, Yi H, Orry A, Kim H et al. Structural and functional plasticity of subcellular tethering, targeting and processing of RPGRIP1 by RPGR isoforms. Biology Open 2012; 1: 140-160.

28. Demirci FY, Rigatti BW, Wen G, Radak AL, Mah TS, Baic CL et al. X-linked cone-rod dystrophy (locus COD1): identification of mutations in RPGR exon ORF15. Am J Hum Genet 2002; 70: 1049-1053.

29. Meindl A, Dry K, Herrmann K, Manson F, Ciccodicola A, Edgar A et al. A gene (RPGR) with homology to the RCC1 guanine nucleotide exchange factor is mutated in X-linked retinitis pigmentosa (RP3). Nat Genet 1996; 13: 35-42.

30. Roepman R, van Duijnhoven G, Rosenberg T, Pinckers AJ, Bleeker-Wagemakers LM Bergen AA et al. Positional cloning of the gene for X-linked retinitis pigmentosa 3 . homology with the guanine-nucleotide-exchange factor RCC1. Hum Mol Genet 1996; 5 : 1035-1041.

31. Vervoort R, Lennon A, Bird AC, Tulloch B, Axton R, Miano MG et al. Mutational hot spot within a new RPGR exon in X-linked retinitis pigmentosa. Nat Genet 2000; 25: 462-466.

32. Otto $\mathrm{E}$, Hoefele J, Ruf R, Mueller AM, Hiller KS, Wolf MT et al. A gene mutated in nephronophthisis and retinitis pigmentosa encodes a novel protein, nephroretinin, conserved in evolution. Am J Hum Genet 2002; 71: 1161-1167.

33. Ayyagari R, Demirci FY, Liu J, Bingham EL, Stringham H, Kakuk LE et al. X-linked recessive atrophic macular degeneration from RPGR mutation. Genomics 2002; 80: 166-171.

34. Mollet G, Salomon R, Gribouval O, Silbermann F, Bacq D, Landthaler G et al. The gene mutated in juvenile nephronophthisis type 4 encodes a novel protein that interacts with nephrocystin. Nat Genet 2002; 32: 300-305.

35. Won J, Gifford E, Smith RS, Yi H, Ferreira PA, Hicks WL et al. RPGRIP1 is essential for normal rod photoreceptor outer segment elaboration and morphogenesis. Hum Mol Genet 2009; 18: 4329-4339.

36. Zhao $Y$, Hong DH, Pawlyk B, Yue G, Adamian M, Grynberg $M$ et al. The retinitis pigmentosa GTPase regulator (RPGR)- interacting protein: Subserving RPGR function and participating in disk morphogenesis. Proc Natl Acad Sci USA 2003; 100: 3965-3970.

37. Lheriteau E, Libeau L, Stieger K, Deschamps JY, Mendes-Madeira A, Provost N et al. The RPGRIP1-deficient dog, a promising canine model for gene therapy. Mol Vis 2009; 15: 349-361.

38. Mellersh CS, Boursnell ME, Pettitt L, Ryder EJ, Holmes NG, Grafham D et al. Canine RPGRIP1 mutation establishes cone-rod dystrophy in miniature longhaired dachshunds as a homologue of human Leber congenital amaurosis. Genomics 2006; 88: 293-301.

39. Chang B, Khanna H, Hawes N, Jimeno D, He S, Lillo C et al. In-frame deletion in a novel centrosomal/ciliary protein CEP290/NPHP6 perturbs its interaction with RPGR and results in early-onset retinal degeneration in the rd16 mouse. Hum Mol Genet 2006; 15: $1847-1857$.

40. Otto EA, Hurd TW, Airik R, Chaki M, Zhou W, Stoetzel C et al. Candidate exome capture identifies mutation of SDCCAG8 as the cause of a retinal-renal ciliopathy. Nat Genet 2010; 42: 840-850.

41. Otto EA, Loeys B, Khanna H, Hellemans J, Sudbrak R, Fan S et al. Nephrocystin-5, a ciliary IQ domain protein, is mutated in Senior-Loken syndrome and interacts with RPGR and calmodulin. Nat Genet 2005; 37: 282-288.

42. Giess A, Trojan P, Rausch S, Pulvermuller A, Wolfrum U. Centrins gatekeepers for the light-dependent translocation of transducin through the photoreceptor cell connecting cilium. Vision Res 2006; 46: 4502-4509.

43. Jauregui AR, Nguyen KC, Hall DH, Barr MM. The Caenorhabditis elegans nephrocystins act as global modifiers of cilium structure. J Cell Biol 2008; 180: 973-988.

44. Jeon CJ, Strettoi E, Masland RH. The major cell populations of the mouse retina. J Neurosc 1998; 18: 8936-8946.

45. Strettoi $\mathrm{E}$, Masland $\mathrm{RH}$. The number of unidentified amacrine cells in the mammalian retina. Proc Natl Acad Sci USA 1996; 93: 14906-14911.

46. Goldberg JL, Klassen MP, Hua Y, Barres BA. Amacrine-signaled loss of intrinsic axon growth ability by retinal ganglion cells. Science 2002; 296: 1860-1864.

47. Lu X, Guruju M, Oswald J, Ferreira PA. Limited proteolysis differentially modulates the stability and subcellular localization of domains of RPGRIP1 that are distinctly affected by mutations in Leber's congenital amaurosis. Hum Mol Genet 2005; 14: 1327-1340.

48. Ni M, Zhou H, Wey S, Baumeister P, Lee AS. Regulation of PERK signaling and leukemic cell survival by a novel cytosolic isoform of the UPR regulator GRP78/BiP. PLOS ONE 2009; 4: e6868.

49. Wang M, Wey S, Zhang Y, Ye R, Lee AS. Role of the unfolded protein response regulator GRP78/BiP in development, cancer, and neurological disorders. Antioxid Redox Signal 2009; 11: 2307-2316.

50. High S, Lecomte FJ, Russell SJ, Abell BM, Oliver JD. Glycoprotein folding in the endoplasmic reticulum: a tale of three chaperones? FEBS Lett 2000; 476: 38-41.

51. Nir I, Papermaster DS. Immunocytochemical localization of opsin in degenerating photoreceptors of RCS rats and rd and rds mice. Prog Clin Biol Res 1989; 314: 251-264.

52. Barnstable CJ. Monoclonal antibodies which recognize different cell types in the rat retina. Nature 1980; 286: 231-235.

53. Sharma N, Kosan ZA, Stallworth JE, Berbari NF, Yoder BK. Soluble levels of cytosolic tubulin regulate ciliary length control. Mol Biol Cell 2011; 22: 806-816.

54. Hammond JW, Huang CF, Kaech S, Jacobson C, Banker G, Verhey KJ. Posttranslational modifications of tubulin and the polarized transport of kinesin-1 in neurons. Mol Biol Cell 2010; 21: 572-583.

55. d'Ydewalle C, Krishnan J, Chiheb DM, Van Damme P, Irobi J, Kozikowski AP et al. HDAC6 inhibitors reverse axonal loss in a mouse model of mutant HSPB1-induced Charcot-Marie-Tooth disease. Nat Med 2011; 17: 968-974.

56. Gardiner J, Barton D, Marc J, Overall R. Potential role of tubulin acetylation and microtubule-based protein trafficking in familial dysautonomia. Traffic 2007; 8: 1145-1149.

57. Dompierre JP, Godin JD, Charrin BC, Cordelieres FP, King SJ, Humbert S et al. Histone deacetylase 6 inhibition compensates for the transport deficit in Huntington's disease by increasing tubulin acetylation. J Neurosc 2007; 27: 3571-3583.

58. Mavlyutov TA, Cai Y, Ferreira PA. Identification of RanBP2- and Kinesin-mediated transport pathways with restricted neuronal and subcellular localization. Traffic 2002; 3: 630-640.

59. Cho KI, Yi H, Yeh A, Tserentsoodol N, Cuadrado L, Searle K et al. Haploinsufficiency of RanBP2 is neuroprotective against light-elicited and age-dependent degeneration of photoreceptor neurons. Cell Death Differ 2009; 16: 287-297.

60. Hubert T, Vandekerckhove J, Gettemans J. Exo70-mediated recruitment of nucleoporin Nup62 at the leading edge of migrating cells is required for cell migration. Traffic 2009; 10 : 1257-1271.

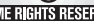

Cell Death and Disease is an open-access journal published by Nature Publishing Group. This work is licensed under the Creative Commons Attribution-NonCommercial-No Derivative Works 3.0 Unported License. To view a copy of this license, visit http://creativecommons.org/licenses/by-nc-nd/3.0/ 